\title{
Crystalline light chain cast nephropathy in multiple myeloma
}

\author{
Zi-Shan Lin $^{1-6, *}$, Xu Zhang ${ }^{1-7,}$, Yi-Yi Ma ${ }^{1-6}$, Su-Xia Wang ${ }^{1-7}$, and Fu-De Zhou ${ }^{1-6}$
}

\begin{abstract}
${ }^{1}$ Renal Division, Department of Medicine, Peking University First Hospital, Beijing; ${ }^{2}$ Institute of Nephrology, Peking University, Beijing; ${ }^{3}$ Renal Pathology Center, Institute of Nephrology, Peking University, Beijing; ${ }^{4}$ Key Laboratory of Renal Disease, Ministry of Health of China, Beijing; ${ }^{5}$ Key Laboratory of CKD Prevention and Treatment, Ministry of Education of China, Beijing; ${ }^{6}$ Research Units of Diagnosis and Treatment of Immune-mediated Kidney Diseases, Chinese Academy of Medical Sciences, Beijing; ${ }^{7}$ Laboratory of Electron Microscopy, Pathological Centre, Peking University First Hospital, Beijing, China
\end{abstract}

Received: August 13, 2020 Revised : August 14, 2020 Accepted: August 14, 2020

\section{Correspondence to \\ Fu-de Zhou, M.D. \\ Tel: +86-13501162527 \\ Fax: +86-010-83575694 \\ E-mail: zhoufude1801@vip.sina.com https://orcid.org/0000-0002- 6355-4946

\footnotetext{
*'These authors contributed equally to this work.
}

A 73-year-old man was referred to our hospital because of 2-week history of oliguria. Laboratory examination revealed hemoglobin $118 \mathrm{~g} / \mathrm{L}$, serum creatinine 1,090.72 $\mu \mathrm{mol} / \mathrm{L}$ and serum immunoglobulin free light chains lambda $>$ 3,925 mg/L. Serum immunoelectrophoresis revealed an immunoglobulin A (IgA) lambda monoclonal band. Urinalysis showed $1+$ proteinuria. Bone marrow examination showed $33 \%$ of monoclonal lambda plasma cells.

A kidney biopsy was obtained, showing moderate tubulointerstitial nephritis with large fractured casts that frequently involves distal tubules. The intraluminal casts demonstrated rodlike and rectilinear crystalline structures, which were associated with focal mononuclear/giant cell reaction. These crystals were brightly fuchsinophilic on the trichrome stain, hypereosinophilic on the hematoxylin and eosin stain, and pale on the Periodic acid Schiff-stain (Fig. 1A and 1B). Immunofluorescence examination unmasked monoclonal reaction of the intraluminal crystalline casts for the lambda light chain without reaction for the kappa light chain (Fig. 1C). Ultrastructural evaluation revealed needle-like and rod-shaped intraluminal crystalline inclusions without substructural organization in the kidney tubules (Fig. 1D).

Therefore, the diagnosis of IgA lamb- da multiple myeloma (MM) and crystalline light chain cast nephropathy (LCCN) with lambda light chain restriction were established.

LCCN is associated with high tumor burden, in which the casts are commonly formed within the distal tubular lumens. Crystallization of monoclonal immunoglobulin can damage the proximal tubules by accumulating within the tubular cytoplasm to cause light chain proximal tubulopathy. Occasionally, light chain crystallization occurs intravascularly or within the cytoplasm of mononuclear phagocytes, leading to crystalglobulinaemia or crystal-storing histiocytosis. Crystalline LCCN is a rare but distinctive morphologic variant of LCCN and has seldom been reported. Patients with crystalline LCCN were mainly associated with lambda light chain restriction and progressed rapidly with a poor prognosis.

Our patient received four cycles of chemotherapy with bortezomib-dexamethasone within 1 month after presentation and achieved partial remission, suggesting that early diagnosis and initiation of bortezomib-based regimens may improve the outcome of MM patients with crystalline LCCN.

This study was performed in accordance with the Helsinki Declaration, and approved by the ethics committee of the Peking University First Hospi- 

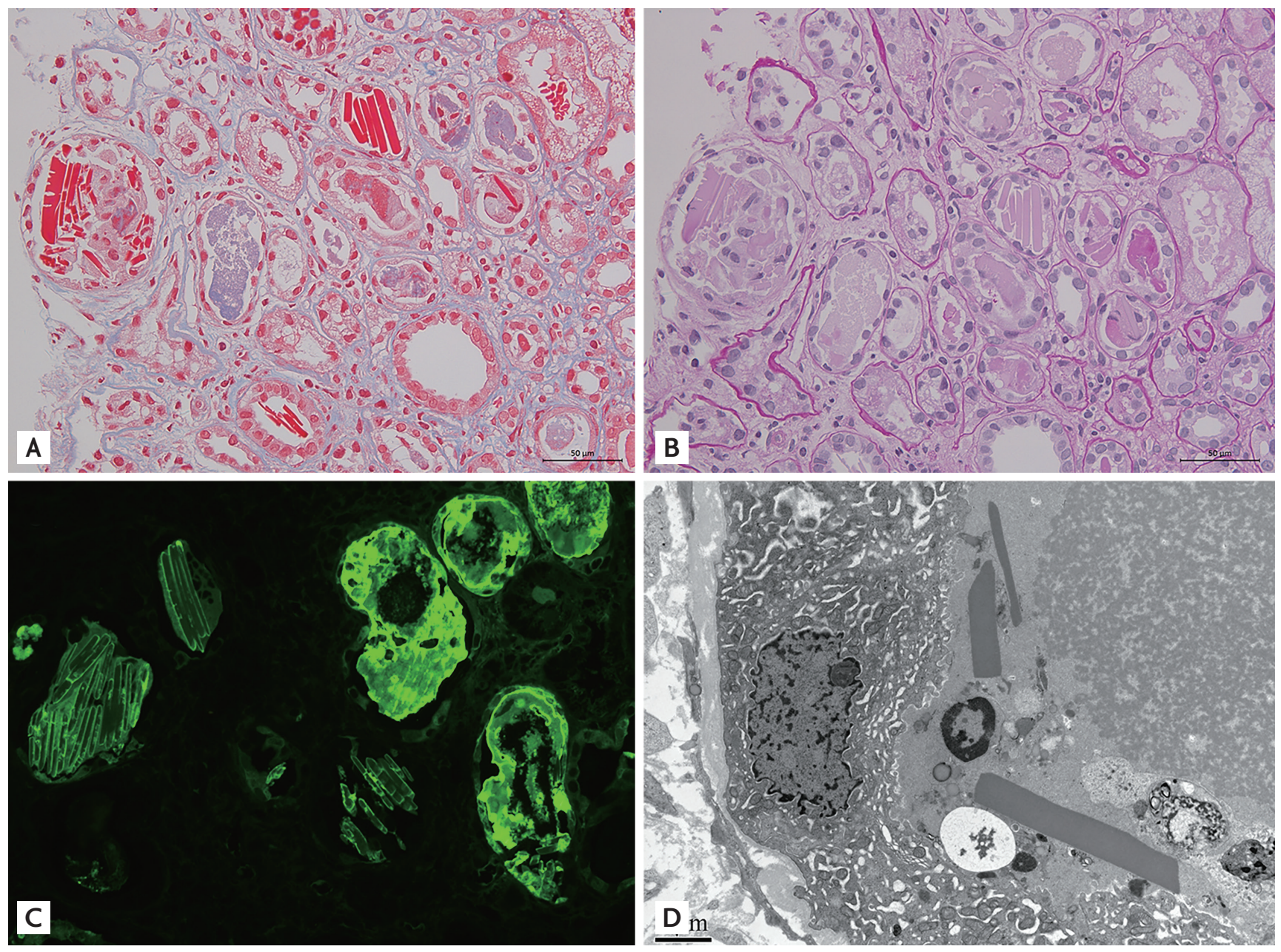

Figure 1. Kidney biopsy findings. (A) Masson staining, original magnification $\times 400$. (B) Periodic acid-Schiff staining, original magnification $\times 400$. (C) Immunofluorescence microscopy for lambda light chain, original magnification $\times 400$. (D) Electron microscopy, original magnification $\times 8,000$.

tal 2017 (1280). Written informed consent were obtained from the patient.

\section{Conflict of interest}

No potential conflict of interest relevant to this article was reported.

\section{Acknowledgments}

This work was supported by the National Natural Sci- ence Foundation of China (Grant Number: 81470956), CAMS Innovation Fund for Medical Sciences (Grant Number: 2019-I2M-5-046) and Scientific Research Seed Fund of Peking University First Hospital (Grant Number: 2018SFoo8). The funders had no role in study design, data collection and analysis, interpretation of the data, decision to publish, or preparation of the manuscript. 